\title{
Deviation from Local Thermodynamic Equilibrium State in Thermal Plasma
}

\author{
Toshihiro KITAMURA, Koichi TAKEDA ${ }^{1)}$ and Kiyoshi SHIBATA ${ }^{2)}$
}

Formerly Nippon Steel Corporation. Now at Interdisciplinary Faculty of Science and Engineering, Shimane University, Nishikawatsu-cho, Matsue, Shimane-ken, 690-8504 Japan.

1) Advanced Materials and Technologies Research Laboratories, Nippon Steel Corporation, Ida, Nakahara-ku, Kawasaki, Kanagawa-ken, 211 -0035 Japan.

2) Formerly Nippon Steel Corporation. Now at Institute for Advanced Materials Processing, Tohoku University, Katahira, Aoba-ku, Sendai, Miyagi-ken, 980-8577 Japan.

(Received on January 5, 1998; accepted in final form on July 6, 1998)

\begin{abstract}
Spectroscopic analysis of $\mathrm{Ar}-\mathrm{H}_{2}$ and $\mathrm{Ar}-\mathrm{N}_{2}$ plasmas revealed that the population density of the excited states of each species ( $\mathrm{Ar}, \mathrm{H}$ and $\mathrm{N}$ ) followed a Boltzmann distribution, but each species had its own respective excitation temperature. The temperature of the argon was lower than that of the hydrogen in the plasma of $\mathrm{Ar}-\mathrm{H}_{2}$ mixture. The temperature of the nitrogen was close to that of the argon in the plasma of $\mathrm{Ar}-\mathrm{N}_{2}$ mixture under one atmospheric pressure. The estimation of the temperatures defined by the velocity distribution functions from the experimental conditions indicate that there are the possibilities to have higher temperatures for the hydrogen than that for the argon in the plasma. These results suggest that there is a deviation from local thermodynamic equilibrium (LTE) in these plasmas under one atmospheric pressure.

The deviations from LTE in these plasmas are explained by the differences in the energy flow in the plasmas. The energy flows of these plasmas are considered as follows from the estimated relaxation times of energy distributions for each species. As for the $\mathrm{Ar}-\mathrm{H}_{2}$ plasma, the electric energy is transferred to the electrons and then the energy of electrons is transferred to the hydrogen atoms. The argon atoms are mainly heated by collisions with the hydrogen atoms. In the case of the $\mathrm{Ar}-\mathrm{N}_{2}$ plasma, both the argon atoms and the nitrogen atoms are heated by collisions with the electrons which obtain the electric energy. As a result, the temperature state of $T_{\theta} \fallingdotseq T_{\mathrm{H}}>T_{\mathrm{Ar}}$ and $T_{\mathrm{e}}>T_{\mathrm{Ar}} \fallingdotseq T_{\mathrm{N}}$ could exist in the plasma.
\end{abstract}

KEY WORDS: thermal plasma; local thermal equilibrium; spectroscopic measurement; Boltzmann plot.

\section{Introduction}

Thermal plasmas which are produced by high current arc and RF induction are of increasing interest for material processing, such as refining, melting and surface treatment. The temperature of a plasma is one of the most important parameters for controlling the process. We can define several temperatures in a plasma, such as $T_{\mathrm{c}}$ for kinetic energy of electrons, $T_{\mathrm{hc}}$ for that of heavy particles, $T_{\mathrm{ex}}$ for population densities of the excited states of every species and $T_{\text {reac }}$ for chemical reactions. Thermal plasma is defined as a plasma in the state of local thermodynamic equilibrium (LTE) where temperatures are the same for all plasma constituents and possible reactions. It is believed that the LTE conditions are usually satisfied in an atmospheric pressure plasma, because of the sufficient collisional interaction among constituent species.

M. M. Kekez ${ }^{1)}$ reported that the temperature of electrons was the same as that of heavy particles in $\mathrm{Hg}$ plasma above the pressure of $3 \times 10^{3} \mathrm{~Pa}$. However it is not always true. H. A. Dinulescu and E. Pfender ${ }^{2}$ predicted by numerical analysis that $T_{\mathrm{e}}$ should be much higher than $T_{\text {he }}$ near electrodes. G. He et $\mathrm{al}^{3}{ }^{3)}$ reported that the difference in the temperature of $\mathrm{Ar}, \mathrm{Fe}$ and $\mathrm{Mn}$ was observed in DC arc plasma under $0.122 \mathrm{MPa}$. Few researchers have investigated the deviation from thermodynamic equilibrium in a thermal plasma under one atmospheric pressure.

The authors will explain the temperature differences between heavy particles in plasmas of $\mathrm{Ar}-\mathrm{H}_{2}$ mixed gas and $\mathrm{Ar}-\mathrm{N}_{2}$ mixed gas produced by a hybrid plasma generator under one atmospheric pressure.

\section{Experimental Apparatus and Procedure}

The experimental apparatus is shown in Fig. 1. It consists of an RF generator $(4 \mathrm{MHz}-80 \mathrm{~kW})$, an DC power supply ( $20 \mathrm{~kW}$ max.), and an optical measuring system. The plasma was formed by a hybrid plasma generator ${ }^{4)}$ which was combined with a DC plasma torch and an induction coupled plasma torch, as shown in Fig. 2. The DC plasma torch consists of a water cooled copper anode and a tungsten cathode. The induction plasma torch is composed of a 3-turn induction coil and a water cooled quartz tube of $60 \mathrm{~mm}$ in inside diameter and $200 \mathrm{~mm}$ in length. The DC torch was operated in a non-transferred mode with $\mathrm{Ar}$ gas. The mixed gas 


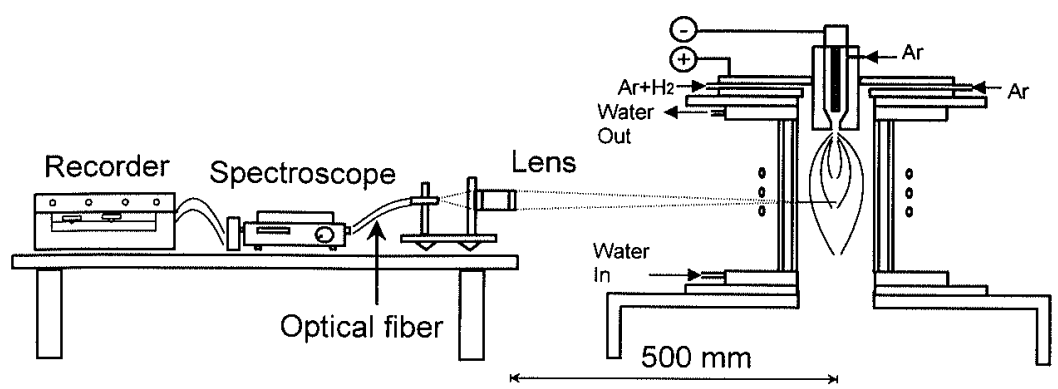

Fig. 1. Experimental arrangement for spectroscopic measurement of plasma.

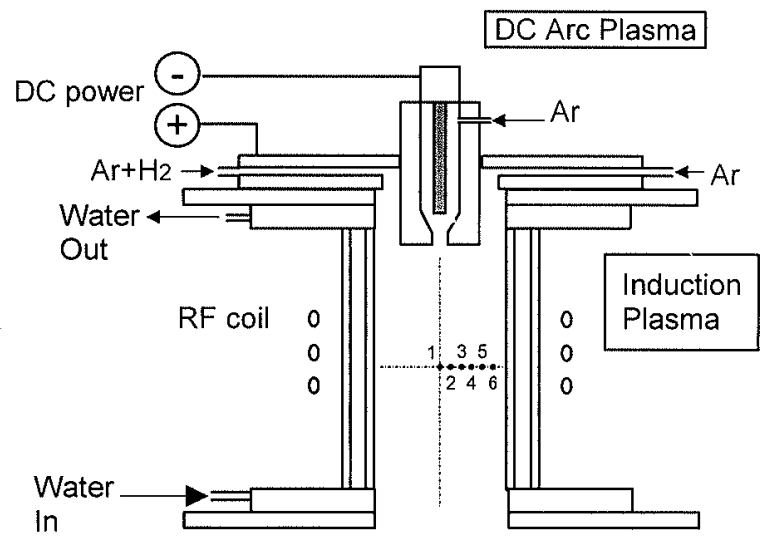

Fig. 2. Schematic diagram of hybrid plasma torch.

Table 1. Experimental conditions.

\begin{tabular}{|c|c|c|c|c|c|}
\hline \multirow[t]{2}{*}{ No } & & & \multicolumn{2}{|c|}{$\begin{array}{c}\text { Gas flow rate } \\
\text { (Nl/min) }\end{array}$} & \multirow{2}{*}{$\begin{array}{c}\text { Power } \\
(\mathrm{kW})\end{array}$} \\
\hline & & & $\mathrm{Ar}$ & $\mathrm{H}_{2}$ or $\mathrm{N}_{2}$ & \\
\hline \multirow{3}{*}{1} & $\mathrm{RF}$ & plasma gas & 25 & $\begin{array}{ll}\mathrm{H}_{2} & 4\end{array}$ & \\
\hline & & sheath gas & 25 & 0 & 64 \\
\hline & $\overline{\mathrm{DC}}$ & plasma gas & 10 & 0 & 4 \\
\hline \multirow{3}{*}{2} & $\mathrm{RF}$ & plasma gas & 25 & $\mathrm{~N}_{2}$ & \\
\hline & & sheath gas & 25 & 0 & 64 \\
\hline & $\mathrm{DC}$ & plasma gas & 10 & 0 & 4 \\
\hline
\end{tabular}

$\left(\mathrm{Ar}+\mathrm{H}_{2}\right.$ or $\left.\mathrm{Ar}+\mathrm{N}_{2}\right)$ was introduced as an $\mathrm{RF}$ working gas through the gap between the anode of the DC torch and the quartz tube. The experimental conditions for the plasma generation are listed in Table 1. All the experiments were carried out under one atmospheric pressure.

The light emitted from the plasma was collected by a lens with a focal length of $105 \mathrm{~mm}$ (NIKON UV105 mm) and transmitted to a spectroscope (NIKON P-250: equipped with a holographic grating blazed at 600 groves $/ \mathrm{mm}$ ) through an optical quartz UV fiber. This optical system was calibrated using a standard Halogen lamp (USHIO $100 \mathrm{~V}-500 \mathrm{~W}$ ).

The intensities of the lights in the lateral direction of the torch were transformed into those in the radial direction by using the Abel inversion technique. ${ }^{5)}$ The validity of the assumption of a Boltzmann distribution was checked by Boltzmann plotting, then the excitation temperatures of constituent species were determined.

\section{Experimental Results}

Figures 3(a) and 3(b) ${ }^{6,7)}$ show the $\ln \left(I_{v} \cdot \lambda / A_{\mathrm{ul}} \cdot g_{\mathrm{u}}\right) v s$. $E_{\mathrm{u}}$ plot for the argon and the hydrogen in the $\mathrm{Ar}-\mathrm{H}_{2}$ mixture plasma which was measured at No. 3 point in
Fig. 2. In the figures, $I_{v}, \lambda, A_{\mathrm{u} 1}, g_{\mathrm{u}}$ and $E_{\mathrm{u}}$ denote the measured spectral intensity of wave number $v$, the wave length of spectra, the transition probability, the statistical weight and the upper energy level, respectively. The values of $\lambda, A_{\mathrm{ul}}, g_{\mathrm{u}}$ and $E_{\mathrm{u}}$ are given in Ref. 8) The excitation temperatures are estimated as $5090 \mathrm{~K}$ for the argon and $12530 \mathrm{~K}$ for the hydrogen from the reciprocal of the slope which was evaluated by the method of least squares. The correlation coefficients are 0.89 for the argon and 0.99 for the hydrogen. Figures 4(a) and $4(b)^{6,7)}$ show those for the argon and the nitrogen in the $\mathrm{Ar}-\mathrm{N}_{2}$ mixture plasma which was measured at No. 3 point in Fig. 2. The excitation temperatures are estimated as $6080 \mathrm{~K}$ for the argon and $4780 \mathrm{~K}$ for the nitrogen from the reciprocal of the slope. The correlation coefficients are 0.91 for the argon and 0.89 for the nitrogen.

Spectroscopic analyses of the plasma revealed that the population density of the excited states of each atomic species ( $\mathrm{Ar}, \mathrm{H}$ and $\mathrm{N}$ ) followed a Boltzmann distribution fairly well as shown in Figs. 3 and 4.

Figure $5^{6,7)}$ shows the temperature profiles of the argon and the hydrogen atoms across the radial direction in the mid-section of the coil, as shown in Fig. 2. The temperature of the hydrogen is higher than that of the argon in the $\mathrm{Ar}-\mathrm{H}_{2}$ plasma. The temperatures of the argon and the nitrogen are shown in Fig. 6, ${ }^{6,7)}$ where the temperatures of both species are close to each other.

\section{Discussion}

Atoms of the same species seem to be in equilibrium, because the populations of the excited atoms at each energy level followed the Boltzmann distribution for each species, as shown in Figs. 3 and 4. The excitation temperature of the hydrogen atoms was much higher than that of the argon atoms. There was a deviation from local thermodynamic equilibrium (LTE) in the $\mathrm{Ar}-\mathrm{H}_{2}$ plasma for the excitation temperatures.

Let us consider the temperature defined by the velocity distribution functions in this experimental conditions. The temperature of monatomic gas in a plasma can be estimated approximately by Eq. (1). ${ }^{9)}$ This equation is derived by the energy balance in the plasma, that is, the exchange of energy by elastic collisions between electrons and monatomic particles is equal to the energy gained by the electrons from the electric field. 


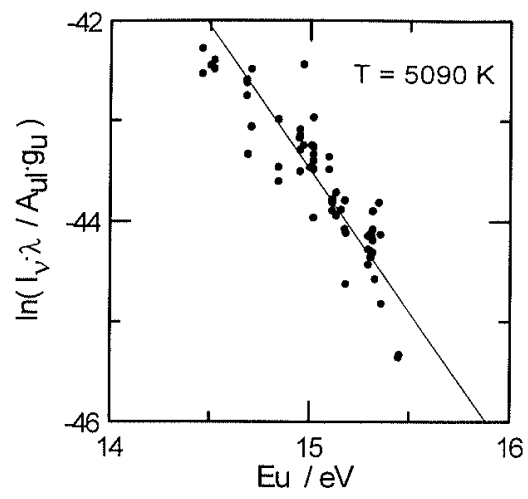

(a) Ar in $\mathrm{Ar}-\mathrm{H}_{2}$ plasma

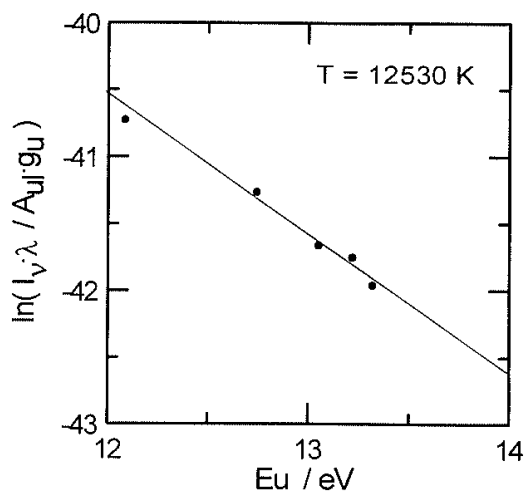

(b) $\mathrm{H}$ in $\mathrm{Ar}-\mathrm{H}_{2}$ plasma

Fig. 3. Atomic Boltzmann plots for $\mathrm{Ar}$ and $\mathrm{H}$ in $\mathrm{Ar}-\mathrm{H}_{2}$ plasma.

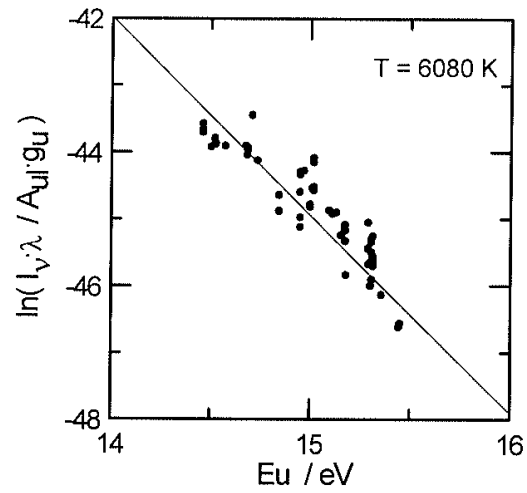

(a) Ar in $\mathrm{Ar}-\mathrm{N}_{2}$ plasma

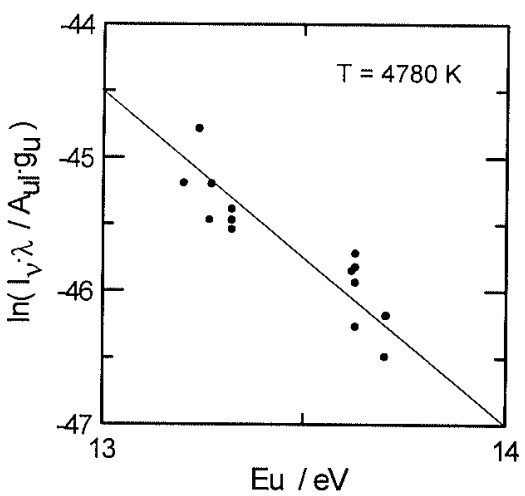

(b) $\mathrm{N}$ in $\mathrm{Ar}-\mathrm{N}_{2}$ plasma

Fig. 4. Atomic Boltzmann plots for $\mathrm{Ar}$ and $\mathrm{N}$ in $\mathrm{Ar}-\mathrm{N}_{2}$ plasma.

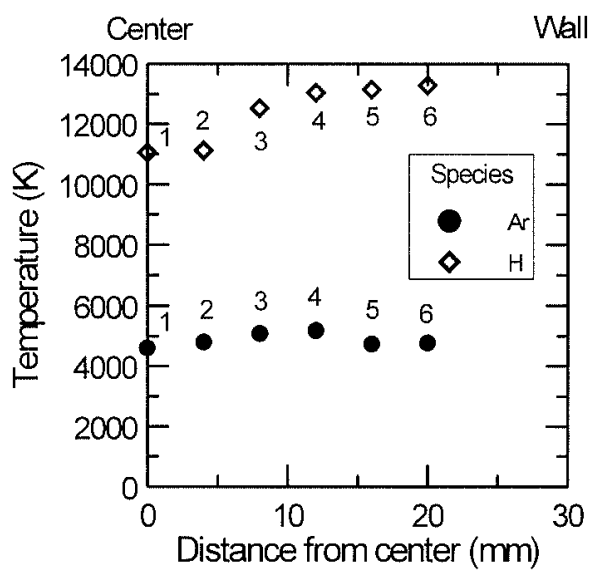

Fig. 5. Temperature profiles of $\mathrm{Ar}-\mathrm{H}_{2}$ plasma in radial direction.

$$
\begin{aligned}
& \frac{T_{\mathrm{e}}-T_{\mathrm{h}}}{T_{\mathrm{e}}}=\frac{m_{\mathrm{h}}}{q m_{\mathrm{e}}}\left(\frac{e \cdot l_{\mathrm{f}} \cdot E}{\kappa \cdot T_{\mathrm{e}}}\right)^{2} \\
& q=24 / \pi
\end{aligned}
$$

where $T_{\mathrm{e}}$ and $T_{\mathrm{h}}$ are the temperatures defined by the velocity distribution functions of electrons and monatomic particles respectively. $m_{\mathrm{e}}$ and $m_{\mathrm{h}}$ are the masses of electrons and monatomic particles respectively. $e, l_{f}$, $E$ and $\kappa$ represent electric charge of an electron, mean free path of an electron, electric field intensity and Boltzmann constant, respectively.

The data of the mean free path, the electron tempera-

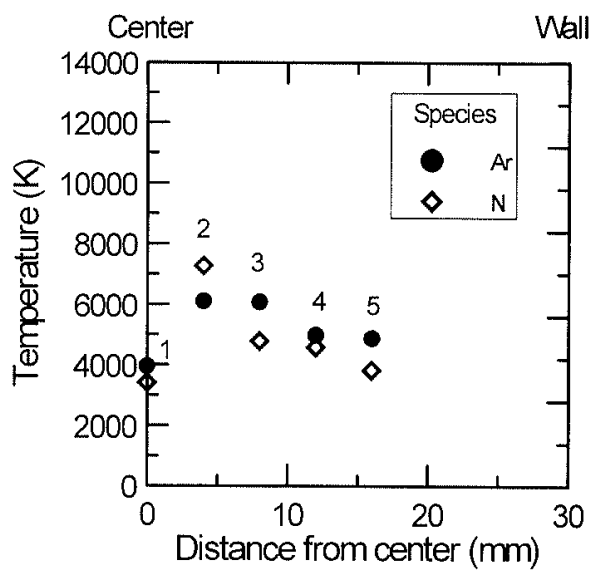

Fig. 6. Temperature profiles of $\mathrm{Ar}-\mathrm{N}_{2}$ plasma in radial direction.

ture and the electric field intensity are needed for the estimation of the temperature of monatomic gas species.

The mean free path of electrons depends on the velocity and species with which they collide, because of the quantum effect. The collisional cross sections are very different from those calculated by the simple solid-solid collision model. Therefore, the mean free paths in this experimental conditions are estimated as $8.3 \times 10^{-5} \mathrm{~m}$ from the data in Refs. 10), 11) for the argon, $1.3 \times$ $10^{-4} \mathrm{~m}$ from the data of Ref. 12) for the nitrogen and $7.0 \times 10^{-5} \mathrm{~m}$ from the data of Ref. 13) for the hydrogen.

The electron temperature could be highest in all species 
Table 2. The estimated temperature of $\mathrm{Ar}, \mathrm{N}$ and $\mathrm{H}$ for several electric field intensities.

$\left(\mathrm{T}_{\mathrm{c}}=14000 \mathrm{~K}\right.$

\begin{tabular}{|c|c|c|c|}
\hline $\begin{array}{c}\text { Electric field intensity } \\
(\mathrm{V} / \mathrm{m})\end{array}$ & $\begin{array}{c}\mathrm{T}_{\mathrm{Ar}} \\
(\mathrm{K})\end{array}$ & $\begin{array}{c}\mathrm{T}_{\mathrm{N}} \\
(\mathrm{K})\end{array}$ & $\begin{array}{c}\mathrm{T}_{\mathrm{H}} \\
(\mathrm{K})\end{array}$ \\
\hline 10 & 13937 & 13942 & 13997 \\
\hline 50 & 12422 & 12563 & 13943 \\
\hline 100 & 7689 & 8252 & 13775 \\
\hline 120 & 4912 & 5723 & 13676 \\
\hline 140 & 1630 & 2735 & 13560 \\
\hline
\end{tabular}

in a plasma. The highest excitation temperature observed in this experiment was $13600 \mathrm{~K}$ for the hydrogen. Although the temperature defined by the velocity distribution functions might be different from the excitation temperature, the electron temperature is assumed to be $14000 \mathrm{~K}$.

The available data of electric field intensity are not enough. The estimated temperatures of the argon, the nitrogen and the hydrogen are shown in Table 2 for several electric field intensities. The higher the electric field intensity, the larger the difference between electron and gas temperature, especially for the argon and the nitrogen. For reference of the intensity, Eckert ${ }^{14)}$ reported the electric field intensity as $50-450 \mathrm{~V} / \mathrm{m}$ depending on the distance from axis $(3-7 \mathrm{~cm})$ for $\mathrm{Ar}$ plasma by RF induction of $2.6 \mathrm{MHz}$. Although it is difficult to compare the reported data with those of this experiment as the radio frequency and dimensions of the torch in the report are different from those in this experiment, the electric field intensity of from 10 to $140 \mathrm{~V} / \mathrm{m}$ in this experiment is not a strange value for a $\mathrm{RF}$ induced plasma.

As a result, the difference in the temperatures defined by the velocity distribution functions could exist in this experimental conditions as shown in Table 2 .

Let us consider the energy flow in the $\mathrm{Ar}-\mathrm{H}_{2}$ and the $\mathrm{Ar}-\mathrm{N}_{2}$ plasmas. In the center region of the RF coil, the molecules of hydrogen and nitrogen are considered to be completely dissociated into atoms. Thus their number density at $5500 \mathrm{~K}$, which is assumed from the excitation temperature of the argon, is determined from their gas flow rates. The fraction of the energy transferred is described to be $\left(2 m_{1} \cdot m_{2}\right) /\left(m_{1}+m_{2}\right)^{2}$ in the case of the collision of two particles with the mass $m_{1}$ and $m_{2}$. The relaxation time of energy transfer by collision, which is the measure of the energy flow between 1 and 2 species, is as follows.

$$
\tau(1-2)=\frac{\left(m_{1}+m_{2}\right)^{2}}{\left(2 m_{1} \cdot m_{2}\right) \cdot\left(v_{1} \cdot N_{2} \cdot \sigma_{1}\right)}
$$

where $v_{1}$ is the velocity of species $1, N_{2}$ is the density of species 2 and $\sigma_{1}$ is the collisional cross section of species 1 for species 2 .

While the time necessary to rearrange the energy distribution among electrons is estimated by the relaxation time ${ }^{15)}$ which is defined as

$$
\tau(\mathrm{e}-\mathrm{e})=\frac{m_{\mathrm{e}}^{1 / 2} \cdot\left(3 \cdot \kappa \cdot T_{\mathrm{e}}\right)^{3 / 2}}{8 \times 0.714 \cdot \pi \cdot N_{\mathrm{c}} \cdot e^{4} \cdot \ln A}
$$

where $\ln A$ is nearly equal to one in these experiments. The electron number density $N_{\mathrm{c}}$ is determined as about
Table 3. The relaxation time of energy transfer for various collisions.

\begin{tabular}{|llll|}
\hline$\tau(\mathrm{e}-\mathrm{e})=4 \times 10^{-8}$ & $\mathrm{sec}$ & $\tau(\mathrm{H}-\mathrm{Ar})=7 \times 10^{-7}$ & $\mathrm{sec}$ \\
\hline$\tau(\mathrm{e}-\mathrm{Ar})=4 \times 10^{-6}$ & $\mathrm{sec}$ & $\tau(\mathrm{N}-\mathrm{Ar})=7 \times 10^{-8}$ & $\mathrm{sec}$ \\
\hline$\tau(\mathrm{e}-\mathrm{N})=1 \times 10^{-6}$ & $\mathrm{sec}$ & $\tau(\mathrm{H}-\mathrm{H})=2 \times 10^{-8}$ & $\mathrm{sec}$ \\
\hline$\tau(\mathrm{e}-\mathrm{H})=5 \times 10^{-8}$ & $\mathrm{sec}$ & $\tau(\mathrm{N}-\mathrm{N})=5 \times 10^{-8}$ & $\mathrm{sec}$ \\
\hline & $\tau(\mathrm{Ar}-\mathrm{Ar})=5 \times 10^{-9}$ & $\mathrm{sec}$ \\
\hline
\end{tabular}

$10^{19} \mathrm{~m}^{-3}$ at $5500 \mathrm{~K}$ by Saha's equation which is assumed thermodynamic equilibrium of $\mathrm{Ar}=\mathrm{Ar}^{+}+\mathrm{e} \cdot{ }^{16)} \mathrm{It}$ is not appropriate strictly to estimate the electron density of a non-equilibrium plasma by Saha's equation. The exciting state of the each species in the plasma is followed Boltzmann distribution in this experiment. Therefore, Saha's equation can be adapted for the rough estimation of the electron density.

The order of $\tau(1-2)$ for various collisions is estimated as shown in Table 3 under this experimental condition. The results of the relaxation time for each species revealed that (1) the energy distribution among electrons could be very quickly, (2) the time necessary for energy transfer from electron to atomic hydrogen is much shorter than that from electron to argon and from electron to atomic nitrogen, and (3) the self relaxation times such as $\tau(\mathrm{Ar}-\mathrm{Ar})$ and $\tau(\mathrm{N}-\mathrm{N})$ are much smaller than the relaxation times of $\tau(\mathrm{e}-\mathrm{Ar})$ and $\tau(\mathrm{e}-\mathrm{N})$. The result of the difference in the relaxation times for each species suggests that the energy flow of the $\mathrm{Ar}-\mathrm{H}_{2}$ plasma is different from that of the $\mathrm{Ar}-\mathrm{N}_{2}$ plasma. As for the $\mathrm{Ar}-\mathrm{H}_{2}$ plasma, the electric energy is transferred to the electrons and they are heated at first, then the energy of the electrons is transferred to the hydrogen atoms. The energy state of the hydrogen atoms is expected to be the same as that of the electrons. The argon atoms were mainly heated by the collisions with the hydrogen atoms, as the energy relaxation time of $\tau(\mathrm{H}-\mathrm{Ar})$ is shorter than that of $\tau(\mathrm{e}-\mathrm{Ar})$. As a result, the energy state of the argon atoms hardly increases. The self relaxation times are small for the electrons, the hydrogen atoms and the argon atoms. Among different species, the temperatures could be expected as follows, $T_{\mathrm{e}} \fallingdotseq T_{\mathrm{H}}>T_{\mathrm{Ar}}$. In the case of the $\mathrm{Ar}-\mathrm{N}_{2}$ plasma, the energy of electrons which is obtained from the electric energy is transferred to both the argon atoms and the nitrogen atoms. The energy relaxation times for $\mathrm{Ar}-\mathrm{Ar}, \mathrm{N}-\mathrm{N}$ and $\mathrm{Ar}-\mathrm{N}$ are shorter than those for e-Ar and e-N. As a result, the temperatures could be expected as follows, $T_{\mathrm{e}}>T_{\mathrm{Ar}} \fallingdotseq T_{\mathrm{N}}$.

One of the reasons of the deviation from LTE in the plasma is considered as the difference in the energy flow in the plasma.The relaxation times themselves are very short, even for e-Ar which is the longest relaxation time in this experiment. Further discussion is needed to know the details of the energy flow in the plasma and to know the details of the relationship between the temperature defined by the velocity distribution functions and the excitation temperature.

The excitation temperature of the argon and the nitrogen increases once and then decreases from the center to the wall of the torch as shown in Figs. 5 and 6 . The tendency of this temperature profiles agree with the calculated results of temperature in a hybrid plasma 
torch by Yoshida et al. ${ }^{17)}$ The temperature of the hydrogen increases from the center to the wall of the torch as shown in Fig. 5. The temperature profiles of the hydrogen could give the proof for the energy flow of electric energy $\rightarrow$ electrons $\rightarrow \mathrm{H} \rightarrow \mathrm{Ar}$ in the plasma discussed above. The energy state of the electron could increase with coming near to the wall of the torch, as the energy is given to the electron by electric energy from outside. As a result, the obtained energy and the temperature of the hydrogen could increase from the center to the wall.

\section{Conclusion}

The validity of the local thermodynamic equilibrium was examined for a plasma generated by the hybrid plasma torch under one atmospheric pressure.

Spectroscopic analysis of $\mathrm{Ar}-\mathrm{H}_{2}$ and $\mathrm{Ar}-\mathrm{N}_{2}$ plasmas revealed that the population density of the excited states of each species (Ar, $\mathrm{H}$ and $\mathrm{N}$ ) followed a Boltzmann distribution, but each species had its own respective temperature. The temperature of the argon was lower than that of the hydrogen in the plasma of $\mathrm{Ar}-\mathrm{H}_{2}$ mixture. The temperature of the nitrogen was close to that of the argon in the plasma of $\mathrm{Ar}-\mathrm{N}_{2}$ mixture under one atmospheric pressure. The estimation of the temperatures defined by the velocity distribution functions from the experimental conditions indicates that there are the possibilities to have different temperatures for each species in the plasma. These results suggest that there is a deviation from LTE in these plasmas under one atmospheric pressure.

The deviations from LTE in these plasmas are explained by the differences in the energy flow in the plasmas. The energy flows of these plasmas are considered as follows from the estimated relaxation times of energy distribu- tions for each species. As for the $\mathrm{Ar}-\mathrm{H}_{2}$ plasma, the electric energy is transferred to the electrons and then the energy of the electrons is transferred to the hydrogen atoms. The argon atoms are mainly heated by collisions with the hydrogen atoms. In the case of the $\mathrm{Ar}-\mathrm{N}_{2}$ plasma, both the argon atoms and the nitrogen atoms are heated by collisions with the electrons which obtain the electric energy. As a result, the temperature state of $T_{\mathrm{e}} \fallingdotseq T_{\mathrm{H}}>T_{\mathrm{Ar}}$ and $T_{\mathrm{e}}>T_{\mathrm{Ar}} \fallingdotseq T_{\mathrm{N}}$ could exist in the plasma.

\section{REFERENCES}

1) M. M. Kekez, M. R. Barraut and J. D. Craggs: J. Phys, D, 13 (1970), 1886.

2) H. A. Dinulescu and E. Pfender: J. Appl. Phys., 51 (1980), 3149.

3) G. He, K. Ishii, Y. Sasaki, Y. Kashiwaya and H. Takahashi: Tetsu-to-Hagané, 82 (1996), 959.

4) T. Yoshida, T. Tani, H. Nishimura and K. Akashi: J. Appl. Phys., 54 (1983), 640

5) O. H. Nestor and H. N. Olsen: SIAM Rev, 2 (1960), 200.

6) T. Kitamura K. Shinbata, K. Takeda and M. Nakamura: Proc. of Japanese Symp. on Plasma Chem., 2 (1989), 257.

7) K. Takada and T. Kitamura: Materia Japan, 33 (1994), 1142.

8) W. L. Wiese, M. W. Smith and B. M. Glennon: Atomic Transition Probabilities, U.S. Dept. of Commerce, NBS, (1966).

9) Plasma Technology in Metallurgical Processing, ed. by J. Feinmann, ISS, (1987), 18.

10) D. E. Golden and H. W. Bandel: Phys. Rev., 149 (1966), 58.

11) M. Mcdowell: Atomic Collision Processes, John Wiley, New York, (1964), 53

12) R. H. Neynaber, L. L. Marino, E. W. Rothe and S. M. Trujillo: Phys. Rev., 124 (1961), 135.

13) R. H. Neynaber, L. L. Marino, E. W. Rothe and S. M. Trujillo: Phys. Rev., 129 (1963), 2069.

14) H. U. Ekert: J. Appl. Phys., 43 (1972), 2707.

15) L. Spitzer, Jr.: Physics of Fully Ionized Gases, John Wiley \& Sons, New York, (1962), 133.

16) A. Kanzawa: Purazumadennetsu, Daigaku Tosyo, Tokyo, (1992), 35 .

17) T. Yoshida, T. Tani, H. Nishimura and K. Akashi: J. Appl. Phys., 54 (1983), 640 